\title{
LE PNEUSOL ET LA MER
}

\author{
N.T. LONG
}

Laboratoire Central des Ponts et Chaussées - 58 boulevard Lefèbvre -

75015 PARIS

\section{ABSTRACT}

Or apart from four artificial islands built for fishes in the Mediterranean sea, some used tires of energy absorption for ships and waves, the utilization of used tires is not common compared with more than two hundred Pneusolstructures in civil engineering.

This paper deals with the different possibilities of this material for the reinforcement of quays, the construction of container platforms, the stabilization of sand based upon experience and engineering know-how.

\section{INTRODUCTION}

A l'exception de quatre récifs artificiels pour des zones de repos pour les poissons en Méditerranée, de quelques pneus utilisés comme amortisseurs pour les bateaux comme pour la houle, lutilisation des pneumatiques usagés est peu répandue dans le domaine du Génie Cottier. Or pendant ce temps, plus de deux cents ouvrages en Pneusol (association de pneus et de sols divers) (Long-Pouget 1978, Cartier-Long 1985, Long 1984) ont été construits dans différents domaines du Génie Civil:

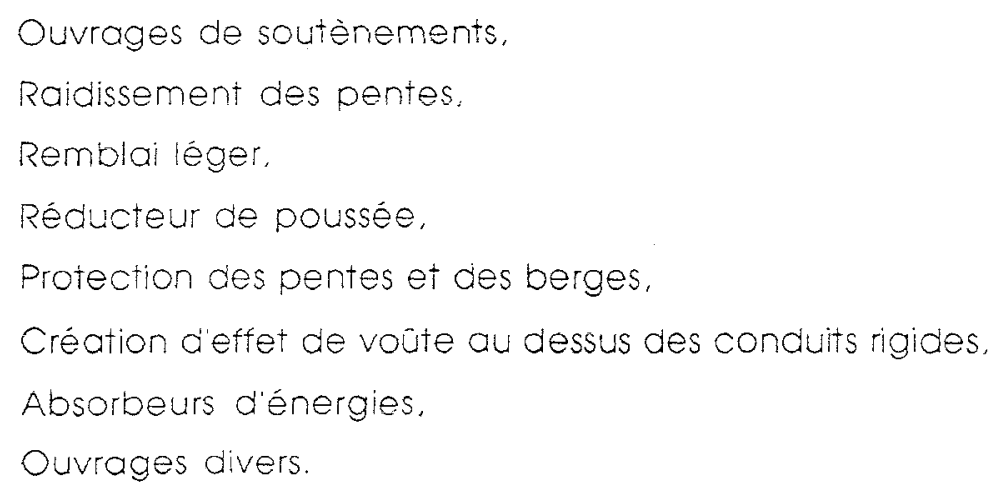

Le bon comportement de ces ouvrages nous incite à regarder vers d'autres applications, en tenant compte de l'expérience acquise dans le domaine du Génie Civil.

En effet, beaucoup de ces réalisations peuvent être adaptées au Génie Côtier car le pneu usagé à un très bon comportement dans l'eau de mer. 


\section{GENERALITES SUR LES PNEUMATIQUES}

Au cours de trois journées d'information (juin 1985), organisées par l'Agence National pour la Récupération ef l'Elimination des Déchets (ANRED), on peut se rendre compte de limportance du gisement de pneus usagés en France qui constitue la plus grande part des déchets de caoutchouc synthétique ou naturel ( 450000 tonnes, avec un taux d'accroissement de $7 \%$ ). $15 \%$ de ces déchets sont récupérés sous des formes diverses:

- Rechapage,

- Sols sportifs.

- L'ensilage

- Brúlage dans les cimenteries...

II reste donc une quantité appréciable qui est jetée dans des décharges contrôlées ou sauvages.

De plus, s'il apparait que les paramètres influençant le vieillissement du pneumatique usagé sont assez nombreux, on peut néanmoins en dénombrer quatre importants:

- la lumiere et la chaleur (les rayons ultra-violets) ont pour effet de provoquer une décomposition surperficielle qui se manifeste par un dessèchement du caoutchouc avec apparition de craquelures plus ou moins profondes. Or le pneu lorsqu'il est visible ne joue pas un rôle mécanique important et de plus il est très fortement armé. La température du sol est trop basse par rapport a celle subie par le pneu lors de son utilisation

- L'ozone : sa présence est faible voire négligeable dans le sol.

- L'acidité du sol : les sols naturels sont relativement peu acides.

Malek et Stevenson (1986) ont mis en évidence son bon comportement dans l'eau de mer en analysant des pneus neufs immergés pendant 42 ans dans la Manche

En conclusion, ces bonnes caractéristiques permettent d'envisager leurs utilisations dans les ouvrages côtiers et maritimes. 


\section{RENFORCEMENT DES MURS DE QUAIS}

De nombreux murs de quais ont subi au cours du temps des problèmes de stabilités dús par exemple à des poussées importantes ou tout simplement au vieillisement de l'ouvrage lui-même. Leur réparation et leur renfocement (injection, palplanches, micro pieux ...) ne posent pas de problème particulier (Levillain 1992). Il existe cependant une solution Pneusol à ces différents problèmes qu'on peut utiliser soit isolément, soit en combinaison avec lune des solutions classiques et qui consiste à réduire voire annuler la poussée horizontale exercée sur le quai.

II suffit pour cela de construire derrière celui-ci, un mur de soutènement ou un Pneusol réducteur de poussée après avoir décaissé le terre-plein. II peut prendre plusieurs formes:

* Soit des nappes de bandes de roulement sur chant liées les unes aux autres et espacées de $0.50 \mathrm{~m}$ comme pour le cas du mur de Mende en Lozère (Figure 1) (Long-pouget 1980. Cartier-Long 1981)

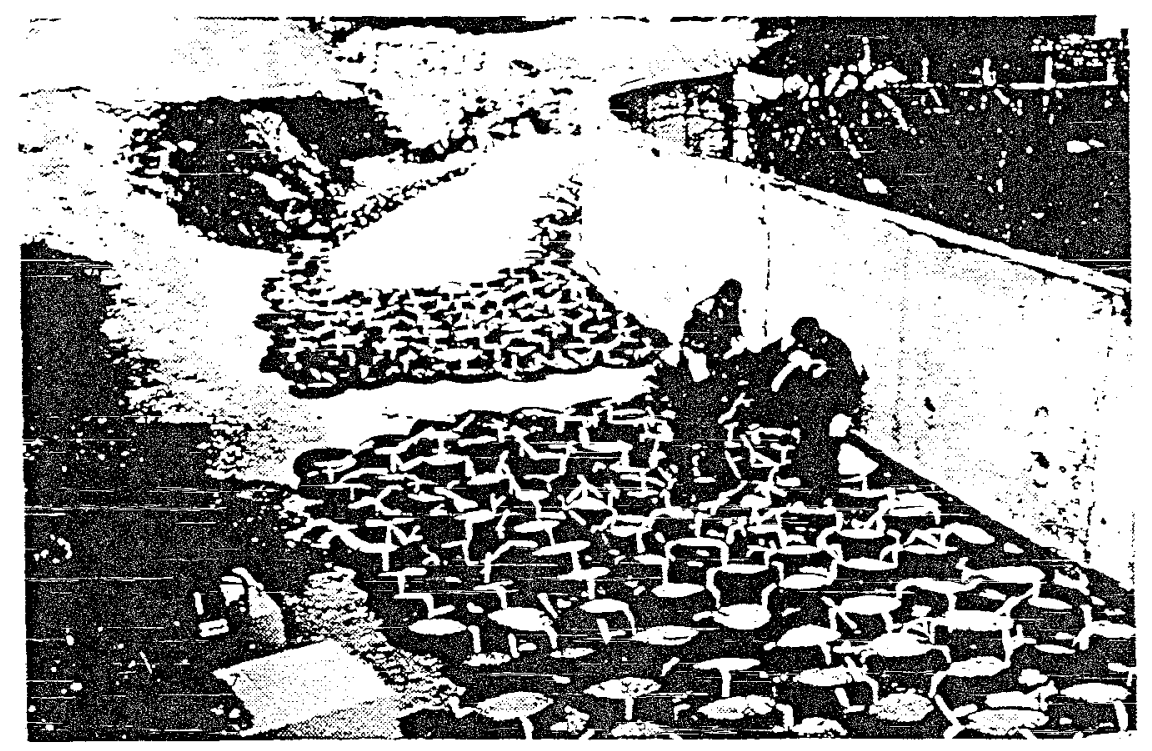

Fig. 1 : Renforcement du mur de Mende

* Soit un ouvrage de soutènement en Pneusol placé juste derrière le quai. II est composé d'un parement en Pneusol constitué de pneus VL ou PL avec un flanc enlevé et de renforcements souples en géotextiles, rigides par exemple en treillis soudés (procédé breveté) ou en Pneusol avec des nappes de bandes de roulement sur chant comme pour le cas précédent (Figure 2).

Ce type de structure pourrait être utilisé, par exemple, pour stabiliser les dunes, car ce parement a la possibilité de récupérer leau de pluie favorisant ainsi une rapide végétalisation. (Long 1984). 
* Soit un Pneusol réducteur de poussée constitué de pneus poids lourds entiers. Les essais sur modèle réduit tridimensionnel de I'INSA de Lyon ont remarquablement mis en évidence cette amélioration. Les "pneus" utilisés sont en mousse de polyuréthane. Ce sont des couronnes de $12 \mathrm{~mm}$. d'épaisseur, et respectivement 30 et $60 \mathrm{~mm}$. de diamètre intérieur et extérieur (échelle de l'ordre $1 / 20^{\circ}$ par rapport à un pneu poids lourd).

Le sol utilisé est un sable concassé de granulométrie uniforme (D. $\mathrm{min}=0.3 \mathrm{~mm}$; D. $\max =1 \mathrm{~mm}$.)

Lo figure n`3 montre le déplacement d'un mur cantilever avec Pneusol et sans Pneusol. L'espacement des nappes étant constant et égate à $18 \mathrm{~mm}$. on fait varier le nombre de pneus par nappe. On constate une très nette amélioration de la stabilité passant de $41 \mathrm{~cm}$. pour $n=0$ (c'est à dire sable seul) à $60 \mathrm{~cm}$. pour $n=4$. Notons de plus que à partir de $n=2$ lo rupture est progressive.

Une heure avant l'ouverture du chantier de l'élargissement de la Nationale menant au Col du Bussang. un mur cyclopéen du lième siècle a été classé comme monument historique (!) par le conservateur en chef d'Alsace. La solution Penuesol utilisée permet de conserver cet ouvrage (fig.4) (Laréal-Long 199!).

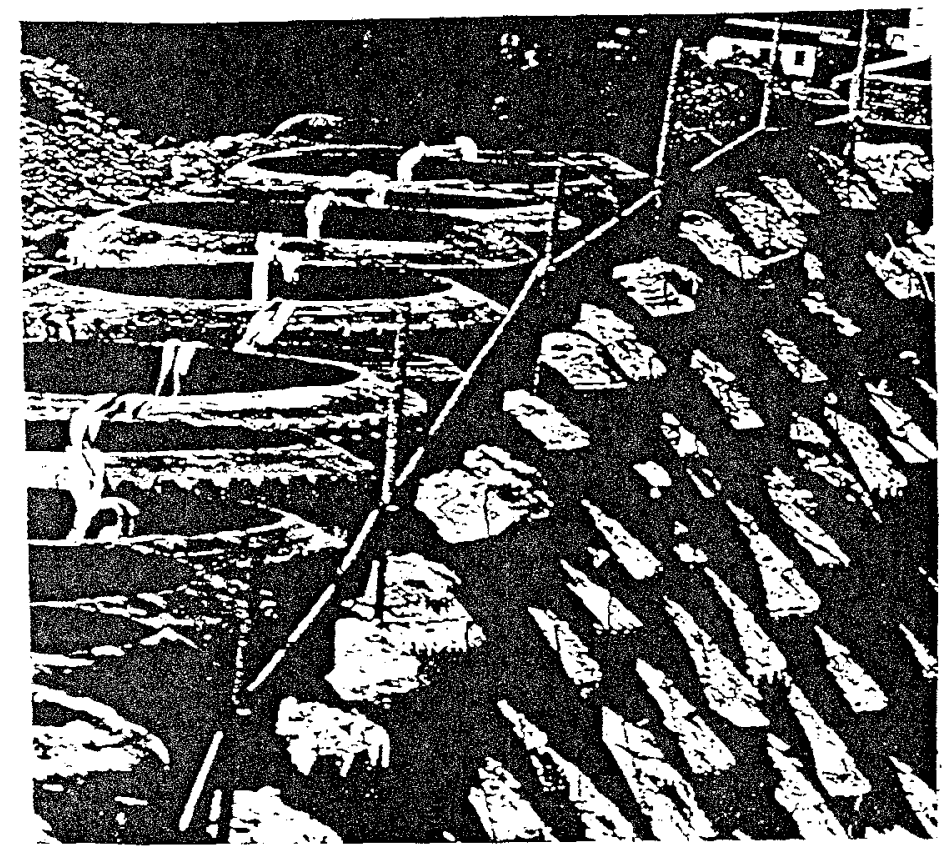

Fig.2 : vue du parement en Pneusol 
spécifications du CFGG pour les géotextiles, les recommandations LCPC-SETRA pour les armatures en acier. En ce qui concerne le Pneusol les méthodes de dimensionnement se trouvent dans les différentes publications et rapports des Laboratoires des Ponts et Chaussées. Le terre-plein qui doit pouvoir supporter une surcharge de lordre de $6 \mathrm{t} / \mathrm{m} 2$, sera traité dans le paragraphe suivant.

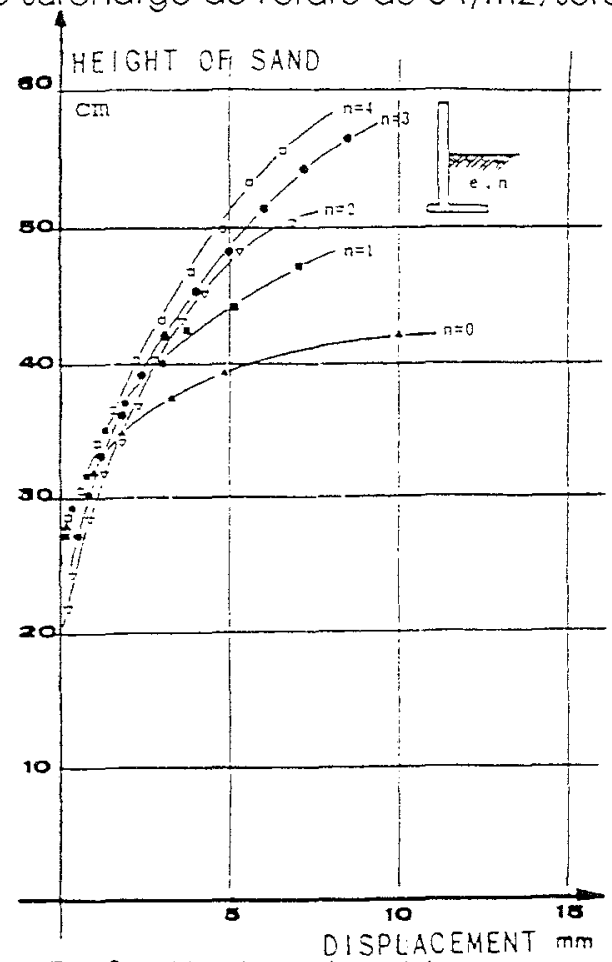

Fig. $\approx$ : Hauteur de sable en

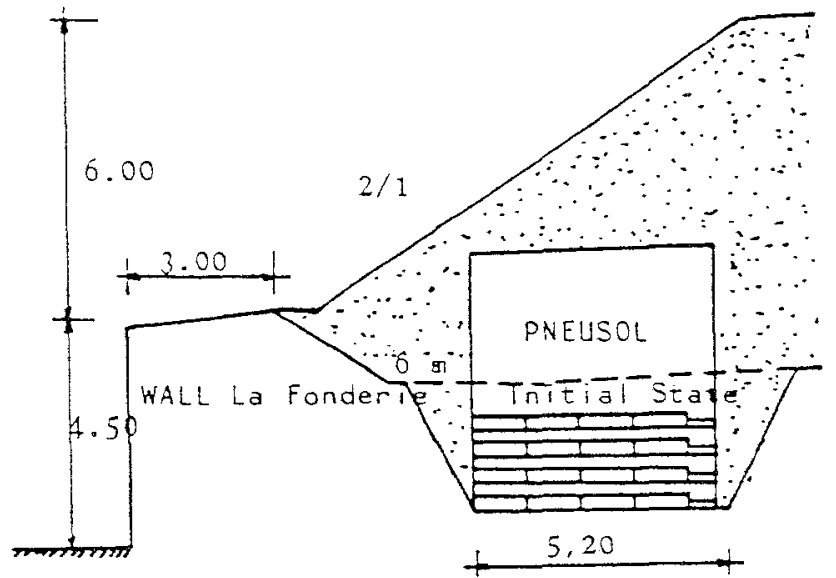

Fig. 4 : Le mur de la Fonderie

fonction du déplacement

\section{CAPACITE PORTANTE DU PNEUSOL}

La capacité portante du matériau dépend pour une grande part du type de Pneusol.

Réalisé avec des bandes de roulement sur chant, celle-cl est identique au sol de remplissage.

Avec des pneus poids lourds disposés en nappe, le remblai rentrant dans le trou de la jante donne au Pneusol un module intermédiaire entre le remblai seul et le pneu seul, avec un coefficient de Poisson faible voir égal à zéro (une extension latérale bloquée). Pour un sable de module dynamique de l'orare de $40 \mathrm{MPa}$ (essai à la dynaplaque) celui du Pneusol sera d'environ 10MPa (soit le module du Limon).

D'une manière générale, lorsqu'on fait un essai de plaque sur un bi-couche un limon surmonté d'une grave. on constate qu'à partir d'une certaine hauteur de grave, linfluence du limon sur le module de l'ensemble a complètement disparu. Cette hauteur est de l'ordre de 50 a $60 \mathrm{~cm}$. C'est pourquoi sur le remblai 
autoroutier de Cannes-Mandelieu, nous avons mis $50 \mathrm{~cm}$ de grave avant de poser les structures de la chaussée (Bailly-Long 1990). Par contre, sur le remblai de Dommiers construit sur un glissement, seulement $35 \mathrm{~cm}$ car la route est départementale et à faible circulation (Bricout et al 1992). Ces deux structures ont été instrumentées et ont montré pour le premier un tassement total de $3 \mathrm{~cm}$ et pour le second un glissement du pied du talus de $2 \mathrm{~cm}$, sans aucune influence sur la chaussée.

Un tel Pneusol était aussi utilisé sur une décharge à Alkirch pour construire trois tennis en 1986 - constitué de trois couches de pneus poids lourds sous $50 \mathrm{~cm}$. de grave propre bien graduée. Six ans après, aucune fissure n'est apparue sur les courts.

En 1987 des essais en vraie grandeur ont été réalisés pour déterminer la capacité portante du sol et du Pneusol par le Laboratoire régional des Ponts et Chaussées de Melun (Canepa) dans une fosse d'environ $230 \mathrm{~m}^{2}$ de surface, $3.20 \mathrm{~m}$ de profondeur, avec une semelle circulaire de $1.60 \mathrm{~m}$ de diamètre. Le sol utilisé est le sable de Fontainebleau et la fosse est remplie d'eau (!). Nous avons ainsi retenu:

- comme pression de rupture gr, la pression correspondant à un enfoncement égal à $15 \%$ de la largeur de la semelle sur la courbe de référence:

- comme fluage caractéristique Ac, la pente des droites de stabilisation corrigées observée sous une "pression de chargement" égale à ar/2 (figure 5)

$\begin{array}{lll}\text { Cas 1 } & \text { ar }=517 \mathrm{kPa} & \mathrm{AC}=6.7 \mathrm{~mm} \\ \text { Cas } 1 & \text { ar }=511 \mathrm{kPa} & \mathrm{AC}=5.4 \mathrm{~mm} \\ \text { Cas } 2 & \text { ar }=247 \mathrm{kPa} & \mathrm{AC}=3.8 \mathrm{~mm} \\ \text { Cas 3 } & \text { ar }=262 \mathrm{kPa} & \mathrm{AC}=1.7 \mathrm{~mm}\end{array}$

Tous ces résultats positifs, associés à l'utilisation de pneus poids lourds très armés (20 a 30\% d'acier de hautes performances), et de bons matériaux de remblais, nous permettent d'envisager le Pneusol comme matériau "antitassement différentiel" en association éventuel avec un géotextile pour la construction de plate-formes de stockage de containers. La figure n'o montre cette utilisation mais seulement sur une couche pour l'élargissement de la Nationale no7 à Cannes-Mandelieu. 


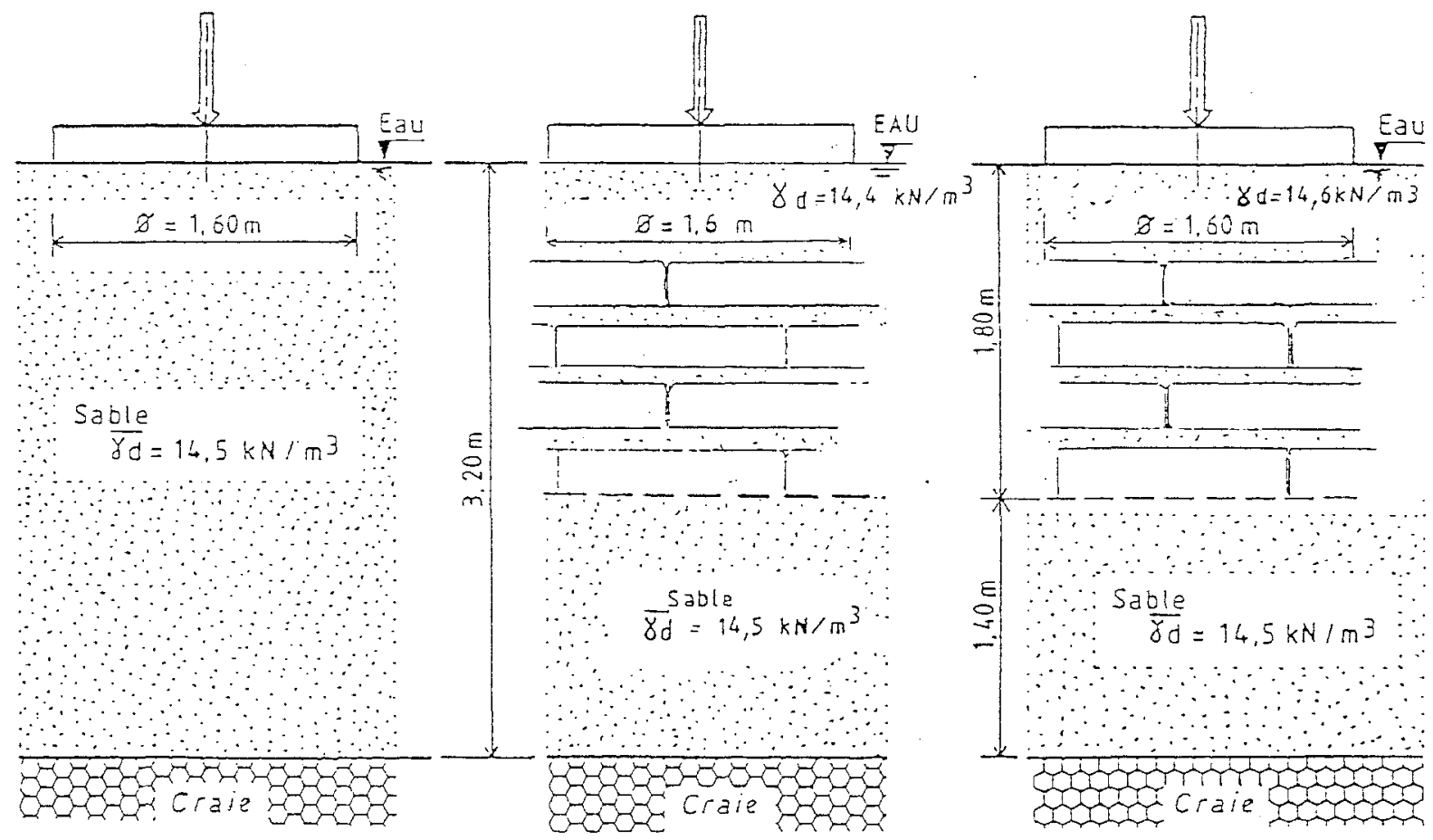

Fig.5: Schéma des différents cas de figures expérimentés

\section{APPLICATIONS DIVERSES}

* Protection des pentes des dunes

Tenant compte de l'expérience de l'Etang du Puits, une digue construite entre 1852 et 1859 et dont la protection mécanique est faite par un Pneusol comportant, en partie supérieure, une nappe de pneus solldarisés entre eux après découpe du flanc supérieur et recouverte de sable. on peut envisager une fixation superficielle des dunes ou des plages par ce type de procédé. La figure nº montre cette protection superficielle à Beaulieu sur Mer contre l'érosion.

\section{* Tapis anti-vibration}

Pour protéger le they de la Grâcieuse des attaques de la Houle, on a construit des épis à laide de barges remplies de sable ef coulées dans la mer qui présentent linconvénlent majeur de disparaitre au bout de quelques années dans le sable. 


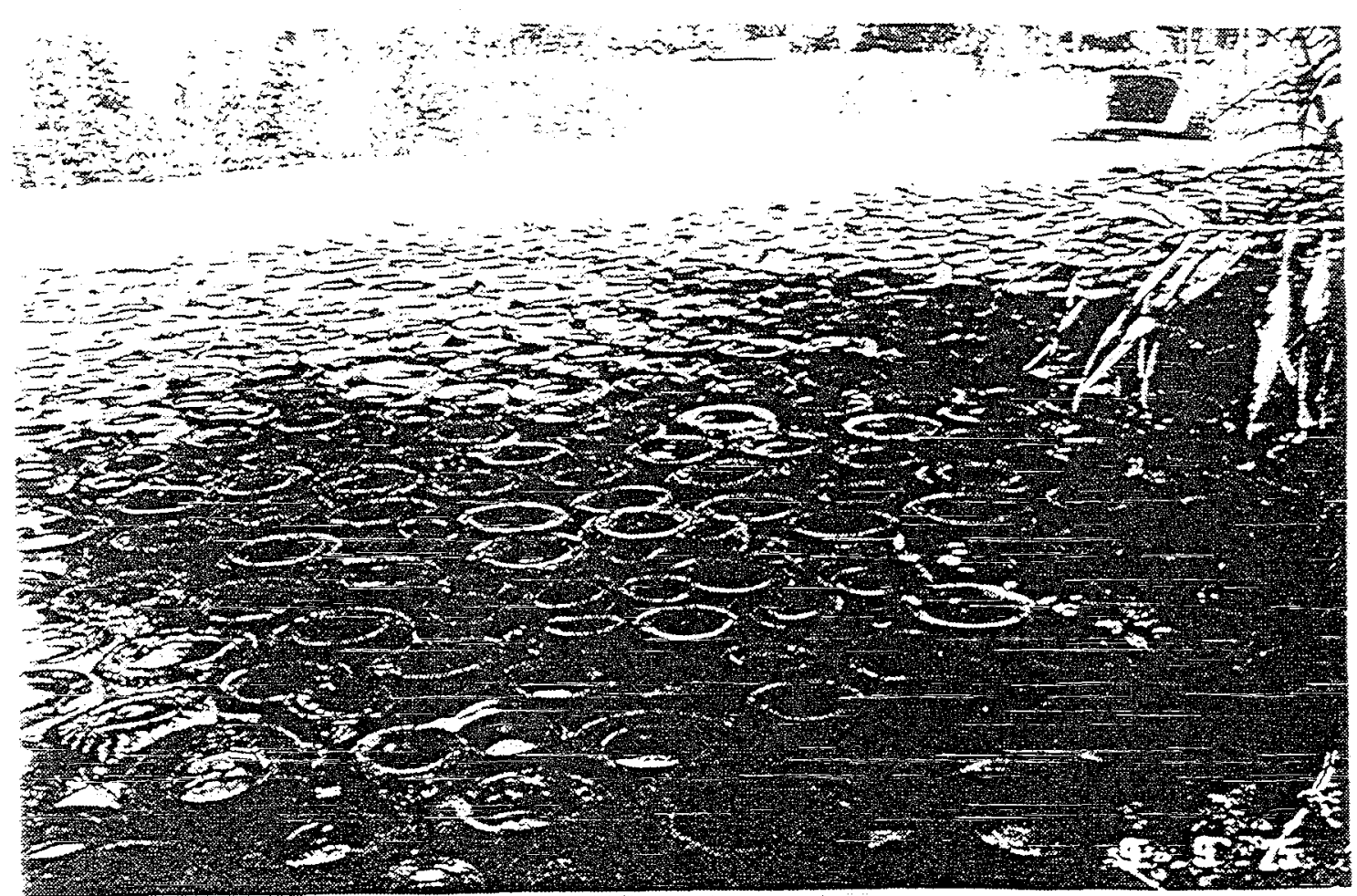

Fig. 6: Vue du chantier de Cannes-Mandelieu

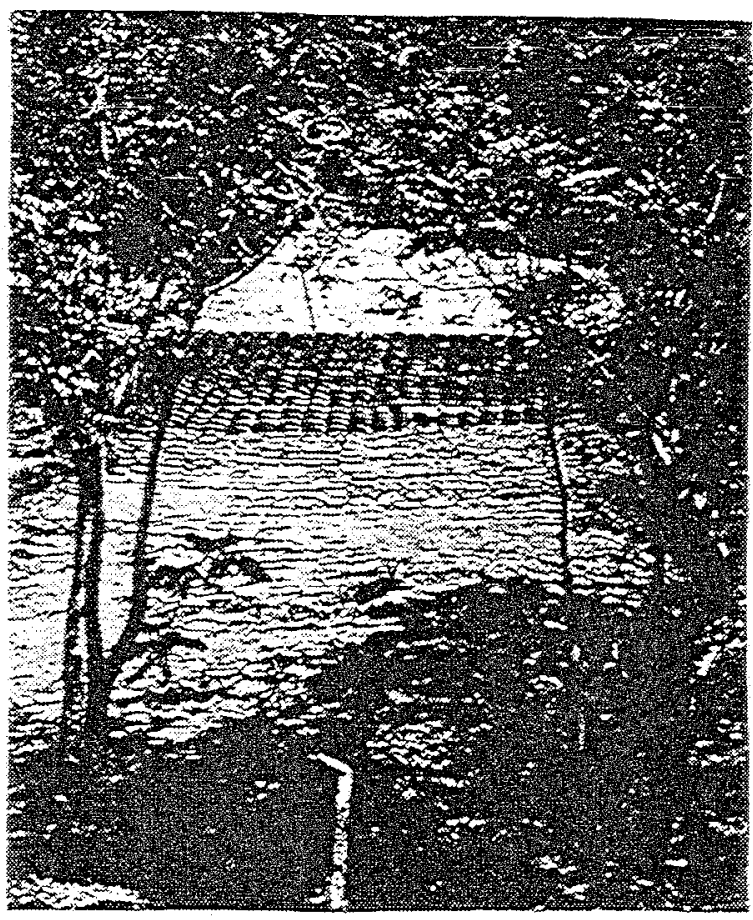

Fig. 7 : Protection superficielle à Beaulieu sur Mer

Pour amortir le balancement et pour mieux répartir les efforts dans le sable. cette barge est coulée sur un lit de Pneusol formé de pneus poids louras 


\section{CONCLUSION}

Les réalisations Pneusol présentées au cours de cette communication ef leur bon comportement montrent bien les possibilités réels de ce matériau dans les domaines maritimes puisqu'actuellement plus de deux cents ouvrages en Génie Civil ont été réalisés en France. Nous n'avons pas abordé toutes ses applications ef notamment le Pneusol répartiteur de contraintes... Ce matériau commence à se développer aux Etat-Unis, en Autriche, en Suisse, en Roumanie, en Algérie, en Espagne, en Italie... La liste de ses possibilités potentelles n'est pas exhaustive.

\section{REFERENCES}

(1) N. T. LONG, P. POUGET, 1980 "Le renforcement des sols par des pneumatiques usagés", Rapport à la DGRST.

(2) G. CARTIER, N.T. LONG, 1991 "Déchets urbains et pneumatiques usagés en Génie Civil", Xème Congrès International de Mécanique des Sols et des Travaux de Fondations, Stockholm.

(3) N.T. LONG, 1984 "Le Pneusol. ", Colloque International sur Routes ef Développement, ENPC, Paris.

(4) K.AB, A. STEVENSON, 1986 "The effect of 42 years immersion in sea water on natural rubber." Journal of Materials Sciences n²1

(5) N. T. LONG (1990) "The Pneusol "Rapport des Laboratoires des Ponts et Chaussées, Série Géotechnique GT 44.

(6) P. Lareal. n.t. LONG, 1991 "Pressure reducing Pneusol: Researchs and Applications", gème Asian Regional Conference on Soll Mechanics and Foundation Engineering. Bangkok.

(7) D. BRICOUT, J.L. DUPRESSOIR, N.T. LONG, 1992 "Réparation d'un glissement par le Pneusol léger", Gème Symposium International sur les glissements de terrain, Christchurch, Nouvelle Zélande.

(8) J.P. LEVILLAIN, 1992 "Rempiètement des murs de quai : quelques techniques". Journées Nationales Génie Côtier-Génie Civil, Nantes. 